\title{
Electron Kinetics Inferred from Observations of Microwave Bursts During Edge Localized Modes in the Mega-Amp Spherical Tokamak
}

\author{
S. J. Freethy, ${ }^{1}$ K. G. McClements, ${ }^{1}$ S. C. Chapman, ${ }^{2,3}$ R. O. Dendy, ${ }^{1,2}$ W. N. Lai, ${ }^{2}$ \\ S. J. P. Pamela, ${ }^{1}$ V. F. Shevchenko, ${ }^{1}$ and R. G. L. Vann ${ }^{4}$ \\ ${ }^{1}$ CCFE, Culham Science Centre, Abingdon OX14 3DB, United Kingdom \\ ${ }^{2}$ Centre for Fusion, Space and Astrophysics, Warwick University, Coventry CV4 7AL, United Kingdom \\ ${ }^{3}$ Max Planck Institute for the Physics of Complex Systems, Nöthnitzer Straße 38, 01187 Dresden, Germany \\ ${ }^{4}$ York Plasma Institute, University of York, York YO10 5DQ, United Kingdom
}

(Received 30 October 2014; published 27 March 2015)

\begin{abstract}
Recent measurements of microwave and x-ray emission during edge localized mode (ELM) activity in tokamak plasmas provide a fresh perspective on ELM physics. It is evident that electron kinetics, which are not incorporated in standard (fluid) models for the instability that drives ELMs, play a key role in the new observations. These effects should be included in future models for ELMs and the ELM cycle. The observed radiative effects paradoxically imply acceleration of electrons parallel to the magnetic field combined with rapid acquisition of perpendicular momentum. It is shown that this paradox can be resolved by the action of the anomalous Doppler instability which enables fast collective radiative relaxation, in the perpendicular direction, of electrons accelerated in the parallel direction by inductive electric fields generated by the initial ELM instability.
\end{abstract}

PACS numbers: 52.35.Hr, 52.35.Qz, 52.55.Fa, 52.55.Tn

The edge localized mode (ELM) [1-3] is a recurring plasma phenomenon driven by steep pressure gradients near the boundary of tokamak plasmas in high confinement $(H$-mode) regimes. A large ELM can deposit $\sim 10 \%$ of the plasma stored energy onto material components. This would be unacceptable in the future burning plasma experiment ITER [4]; it is therefore vital to understand all aspects of the plasma physics of ELMs in order to predict and mitigate their effects in future experiments. The instability that initiates ELMs is relatively well understood at the fluid level of description (see, e.g., Ref. [5]). However, the observations reported here of electromagnetic radiation from plasmas with ELM activity indicate that the physics of this process incorporates electron kinetic effects that are not included in fluid descriptions and occur on relatively short length scales and time scales. For example, in the Mega-Amp Spherical Tokamak (MAST) [6], ELMs are accompanied by intense bursts of microwave emission (BMEs) in the electron cyclotron (EC) frequency range. These can reach brightness temperatures 3-4 orders of magnitude higher than the thermal background, and detailed experimental accounts of this emission have been given by others [7-10], and new observations on MAST using the Synthetic Aperture Microwave Imaging Radiometer [11] are broadly consistent with these accounts.

Published by the American Physical Society under the terms of the Creative Commons Attribution 3.0 License. Further distribution of this work must maintain attribution to the author(s) and the published article's title, journal citation, and DOI.
The peak emission frequency of the BMEs lies above the cyclotron frequency in the outer plasma edge $\Omega_{c \text {,edge }}$, with $\omega / \Omega_{c, \text { edge }} \sim 1.3-1.4$ and with a bandwidth of $\delta \omega / \omega \sim 0.3$. This narrow bandwidth rules out an explanation of the BMEs in terms of highly relativistic electrons, unless some effect causes the emitting electrons to have a narrow range of energies [12]. There is no external heating source on MAST capable of accelerating electrons in the perpendicular direction, so the inferred presence of energetic electrons must be due to an intrinsic plasma effect.

Intense bursts of coherent radiation similar to BMEs in tokamaks are observed in many space and astrophysical plasma environments, including solar flares [13] whose phenomenology may have much in common with that of ELMs [14]. In this Letter we show for the first time that BMEs in MAST can be attributed to the presence of suprathermal, magnetic-field-aligned electron populations that drive waves in the EC range via the anomalous Doppler instability (ADI). This instability has been observed recently on the HT-7 tokamak [15] and is also believed to play a role in the dynamics of energetic electrons in solar flares $[16,17]$ and the excitation of whistlers in the solar wind [18]. The production and evolution of energetic electron populations continue to be of topical interest in the solar context [19]. An electron with velocity parallel to the magnetic field $v_{\|}$is in resonance with a wave of frequency $\omega$ and parallel wave vector $k_{\|}$if

$$
\omega=\ell \Omega_{e}+k_{\|} v_{\|},
$$

where $\ell$ is an integer and $\Omega_{e}$ is the EC frequency. The ADI occurs when waves are excited via this resonance condition 


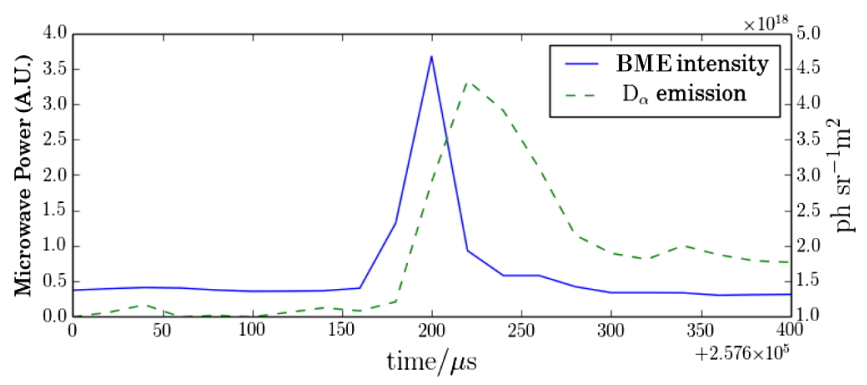

FIG. 1 (color online). Evolution of microwave intensity (solid line) and $D_{\alpha}$ (dashed line) emission in a typical ELM.

with $\ell<0$; in applications of the ADI to tokamak plasmas, the case $\ell=-1$ is generally found to be relevant [20]. As the ADI proceeds, the suprathermal electrons acquire perpendicular momentum comparable to their parallel momentum on time scales of a few hundred EC periods $\tau_{c}$. While predominantly electrostatic, the waves can be converted to electromagnetic waves and thus propagate freely to antennas outside the plasma [21].

BME intensities relative to the average thermal background level peak at about $35 \mathrm{~dB}$ and extend up to $40 \mathrm{~dB}$. The BMEs also correlate in time with individual ELMs, as shown in Fig. 1. The peak of the BME emission typically occurs $20 \mu \mathrm{s}$ before that of the deuterium- $\alpha\left(D_{\alpha}\right)$ line emission, which is the primary diagnostic signature of an ELM and is due to filamentary structures erupting into the relatively cold region outside the confined plasma. In many cases a burst of $\mathrm{x}$-ray emission coincides with the microwave burst, with the peak emission again preceding the $D_{\alpha}$ emission by around $20 \mu$ s in the crash phase [22]. Figure 2 shows an example, obtained using a soft $\mathrm{x}$-ray camera that is sensitive to photons with energies from 1 to $30 \mathrm{keV}$ emitted along many lines of sight crossing the plasma. The fluxes plotted in Fig. 2 were recorded for a line of sight that passes through the plasma low field side edge above the midplane. The peak x-ray intensity is more than double the pre-ELM value. Smaller enhancements in x-ray intensity are seen for lines of sight passing closer to the plasma center.

Radiometer measurements at high time resolution reveal fine structure in the BMEs, as shown in Fig. 3. Individual

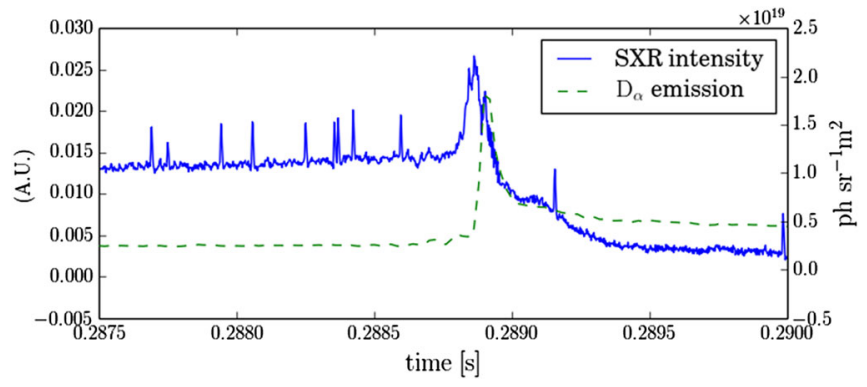

FIG. 2 (color online). Evolution of soft x-ray (SXR) intensity (top) and $D_{\alpha}$ emission (bottom) in a typical ELM.

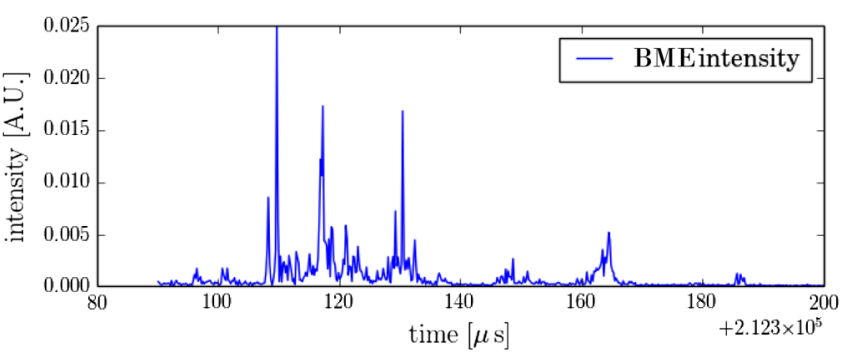

FIG. 3 (color online). Microwave intensity of a BME versus time recorded using a high time resolution radiometer.

bursts have rise and decay times of the order of $1 \mu \mathrm{s}$, and fine structure is also seen in the soft x-ray bursts (Fig. 2).

The near-universal occurrence of the BMEs and the frequent concurrence of soft x-ray bursts during ELMs in MAST are strong evidence for the presence of energetic electrons. A rise in soft $\mathrm{x}$-ray emission could arise from an inflow of impurities into the plasma as a result of the ELM. However, the fact that the peak in the soft $\mathrm{x}$-ray emission precedes that of the $D_{\alpha}$ emission, with the x-ray emission decaying on a time scale of a few tens of microseconds, makes it more likely that the enhanced emission results from nonthermal bremsstrahlung.

While ELMs are likely to be triggered by ideal magnetohydrodynamic instabilities, the later stages of these events, culminating in the detachment of filamentary structures from the main plasma, must involve nonideal effects. Simulations of ELMs in MAST performed with the nonlinear resistive magnetohydrodynamic code JOREK [23] reveal that parallel electric fields of up to $2 \mathrm{kV} \mathrm{m}^{-1}$ can be expected in localized regions (Fig. 4). Mirnov coil measurements during ELMs in MAST imply that the modes associated with these fields have periods of the order of a few microseconds; individual electrons would be accelerated to

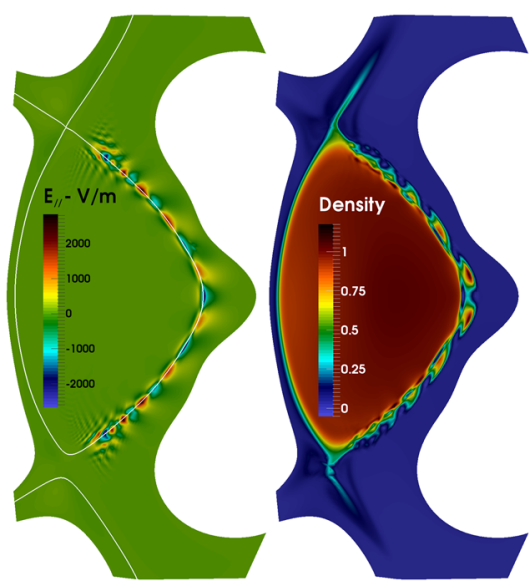

FIG. 4 (color online). Poloidal snapshots of parallel electric field (left plot) and plasma density normalized to the magnetic axis value (right plot) in a JOREK simulation of an ELM in MAST [23]. The white curve in the left plot shows the separatrix. 
tens of $\mathrm{keV}$ if they encountered $2 \mathrm{kV} \mathrm{m}^{-1}$ parallel electric fields for only a small fraction of this time.

Given the presence of magnetic-field-aligned suprathermal electrons, we now demonstrate that they are unstable to the generation of waves in the EC range under MAST-like plasma conditions. To address this, particle-in-cell (PIC) simulations have been carried out in one space dimension and three velocity dimensions, the magnetic field being tilted at an angle of $45^{\circ}$ with respect to the space axis. Thus, any excited waves have wave vectors with components parallel $\left(k_{\|}\right)$and perpendicular $\left(k_{\perp}\right)$ to $\mathbf{B}$ of equal magnitude, i.e., $k_{\|}=k_{\perp}$. Across much of the MAST plasma, due to the high $\beta$, the EC frequency is smaller than the electron plasma frequency $\omega_{p e}$; in conventional tokamaks the opposite inequality generally applies [20]. The location in the MAST plasma of the instability producing the microwave bursts is not precisely known. The close correlation with ELMs suggests that it is likely to be close to the low field side plasma edge, but the electron density typically varies by more than an order of magnitude between the inner edge of the $H$-mode pedestal (steep pressure gradient region) and the plasma edge. For this reason, PIC simulations have been carried out with a range of values of initial bulk electron density $n_{e}$, from $2 \times 10^{18}$ to $4 \times 10^{19} \mathrm{~m}^{-3}$. In all cases the initial electron distribution comprises a Maxwellian bulk with a temperature $T_{e}$ of either 100 or $20 \mathrm{eV}$ and a flat magnetic-field-aligned tail, containing $5 \%$ or $10 \%$ of the total electron population and extending to 20 or 40 times $v_{B}$ where $v_{B}=\left(2 T_{e} / m_{e}\right)^{1 / 2}$ is the initial bulk electron thermal speed, $m_{e}$ being the electron mass. It is known that the growth rate of the ADI is linearly dependent on the fractional density $n_{f} / n_{e}$, so we may extrapolate the measured growth rates to smaller fractional densities if necessary. In all cases the equilibrium magnetic field $B_{0}$ is $0.4 \mathrm{~T}$, a typical value in the outer midplane edge of MAST plasmas.

Figure 5 shows the result of Fourier transforming, in space and time, the electric field component parallel to the wave vector (i.e., the electrostatic component) in a simulation with initial $n_{e}$ equal to $2 \times 10^{18} \mathrm{~m}^{-3}$. Two dominant forward-propagating $(k>0)$ waves are excited by the ADI, with frequencies at large $k$ approximately equal to $0.46 \omega_{p e} \simeq 2 \pi \times 5.8 \mathrm{GHz}$ and $1.2 \omega_{p e} \simeq 2 \pi \times 15.2 \mathrm{GHz}$. The first of these frequencies is below the range detectable using microwave diagnostics on MAST, while the latter agrees well with the observed peak emission frequencies for BMEs on MAST and is also close to the upper hybrid resonance $(\mathrm{UHR})$ frequency $\omega_{\mathrm{UH}}=\left(\omega_{p e}^{2}+\Omega_{e}^{2}\right)^{1 / 2}$ which is around $1.33 \omega_{p e}$ for the parameters used here. Predominantly electrostatic waves in this frequency range can be converted to electromagnetic modes and then detected by antennas outside the plasma.

Figure 6 shows (a) the initial electron velocity distribution in this simulation and (b) the distribution after $379.4 \tau_{c} \simeq 30 \mathrm{~ns}$. It is apparent that, in this short time,

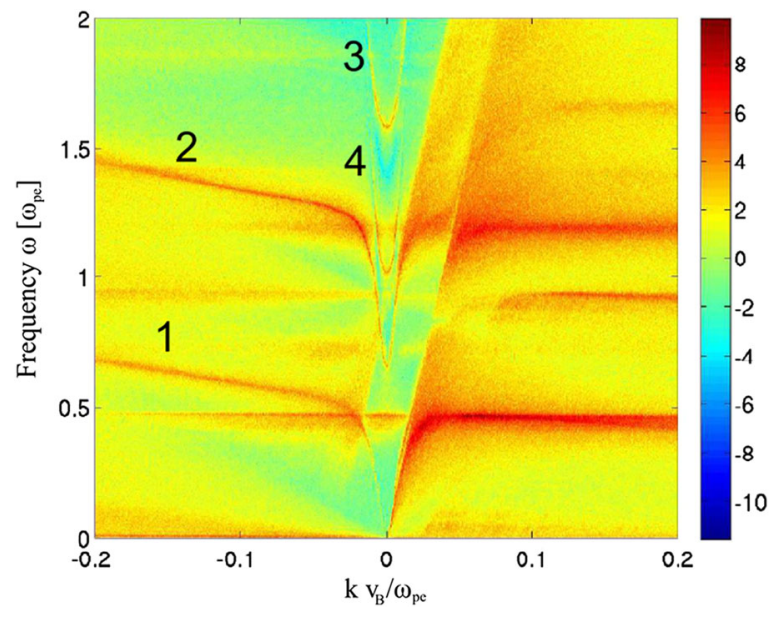

FIG. 5 (color online). Electrostatic field amplitude (on the logarithmic scale) versus wave number and frequency in PIC simulation with initial electron density $2 \times 10^{18} \mathrm{~m}^{-3}$. Branch 1 is a whistler mode, 2 is a generalized Langmuir mode, 3 is an $O$ mode, and 4 is an $X$ mode.

the electrons in the tail acquire perpendicular momenta comparable to their initial parallel momenta. We conclude that strongly field-aligned energetic electron distributions in the edge plasma of MAST are very rapidly isotropized via the ADI. From Fig. 5 we note that the forward-propagating waves in the upper hybrid frequency range have wave vectors $k \sim 0.1 \omega_{p e} / v_{B}$ and hence $k_{\|} \sim 0.1 \omega_{p e} /\left(\sqrt{2} v_{B}\right)$. Using this value of $k_{\|}$in Eq. (1) and setting $\omega=1.2 \omega_{p e}, \ell=-1$, we find that the anomalous Doppler resonance condition is satisfied by electrons with $v_{\|} \sim 30 v_{B}$; we note from Fig. 6 that this is close to the parallel velocity at which electrons have acquired the largest boost in $v_{\perp}$ and conclude from this that the ADI explains the excitation of the high amplitude waves observed in this simulation.

Similar phenomena are observed in PIC simulations with higher $n_{e}\left(\geq 10^{19} \mathrm{~m}^{-3}\right)$ and $T_{e}(100 \mathrm{eV})$, more characteristic of the top of the $H$-mode pedestal region in MAST plasmas. However, as in the low density case discussed above, high amplitude waves are only excited in these
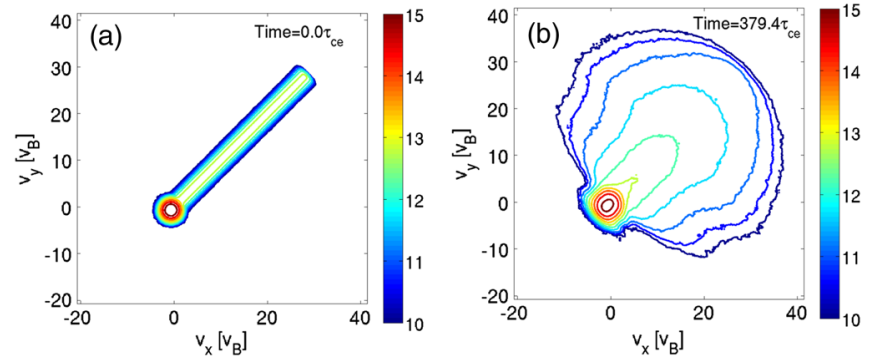

FIG. 6 (color online). Contours of (a) initial and (b) final electron velocity distributions in PIC simulation with initial electron density $2 \times 10^{18} \mathrm{~m}^{-3}$ and a nonthermal electron tail fraction of $5 \%$. The color bar has a logarithmic scale. 
simulations at frequencies either below $\Omega_{e}$ or above $\omega_{p e}$. Neither of these frequency ranges is compatible with the observed frequency peak, if $B_{0}=0.4$ and $n_{e} \geq 10^{19} \mathrm{~m}^{-3}$. Only in the low density simulation do we see wave excitation at frequencies that are consistent with the BME spectra, suggesting that the energetic electrons causing the emission undergo acceleration very close to the MAST plasma edge. This conclusion is bolstered by the predictions from JOREK shown in Fig. 4.

A corollary of this last conclusion is that the energetic electrons are likely to have a very short confinement time, particularly in view of the fact that the magnetic field $\mathbf{B}$ in the plasma edge region is strongly distorted by the ELM instability. In these circumstances we expect the electrons to be subject to rapid cross-field transport of the type discussed by Rechester and Rosenbluth [24]. Generally, the condition $\nabla \cdot \mathbf{B}=0$ implies that a perturbation $\delta \mathbf{B}$ to a magnetic field $\mathbf{B}$ causes the field line to be displaced by $\Delta \sim(\delta B / B) L_{c}$ where $L_{c}$, the parallel length scale of the field, can be taken in tokamaks to be of order $q R$ where $q$ is the safety factor and $R$ is the major radius. This implies a cross-field diffusion rate $D_{e}$ of order $\Delta^{2} / \tau_{\|}$where $\tau_{\|} \sim q R / v_{\|}$, i.e., $D_{e} \sim v_{\|} q R(\delta B / B)^{2}$, and a confinement time $\tau_{e} \sim \delta r^{2} / D_{e}=\delta r^{2}(B / \delta B)^{2} /\left(q R v_{\|}\right)$where $\delta r$ is the radial distance from the acceleration site to the last closed flux surface. Given that $v_{\|}$is likely to be of order $10^{8} \mathrm{~ms}^{-1}$, while $R \simeq 1 \mathrm{~m}, q \gg 1$, and $\delta r \sim 1 \mathrm{~cm}$, it is evident that confinement times of the order of microseconds, consistent with the rapid burst time scales apparent in Fig. 3, could result from rather modest field perturbations, e.g., $\delta B / B \sim 10^{-4}$.

We consider finally the soft $\mathrm{x}$-ray data. It is reasonable to suppose that the preburst emission is dominated by thermal bremsstrahlung, while the emission during the burst consists largely of nonthermal bremsstrahlung produced by energetic electrons. Using the simplest approximation to the bremsstrahlung cross section [25], assuming that the energetic electrons have a flat tail distribution of the type used in the PIC simulations and integrating the bremsstrahlung energy flux over photon energies above the soft x-ray camera threshold $\mathcal{E}_{0}$, the ratio of nonthermal to thermal emission from a homogeneous region of plasma is given by

$$
\frac{\mathcal{F}_{\mathrm{NT}}}{\mathcal{F}_{\mathrm{T}}}=\frac{\pi^{1 / 2}}{3} \frac{n_{f}}{n_{e}}\left(\frac{E_{\mathrm{max}}}{T_{e}}\right)^{1 / 2} e^{\mathcal{E}_{0} / T_{e}},
$$

where $n_{f}$ and $E_{\max }$ are the density and maximum energy of the energetic electrons. As discussed previously, Fig. 2 shows fluxes of x-rays emitted along a chord passing through the plasma edge region, where $T_{e}$ is at most $100-200 \mathrm{eV}$. Since $\mathcal{E}_{0} \simeq 1 \mathrm{keV}$, the exponential factor in Eq. (2) is much larger than unity and, if we assume also that $E_{\max }$ lies in the tens of $\mathrm{keV}$ range, it follows that a relatively modest energetic electron fraction $n_{f} / n_{e}$ (typically $10^{-3}$ or less) would be sufficient to produce the enhancement in $\mathrm{x}$-ray intensity apparent in Fig. 2. The inference that only a small fraction of the local electron population is accelerated during an ELM is reinforced by Thomson scattering measurements of $T_{e}$. Large transient rises in the edge plasma $T_{e}$ have not been detected when Thomson scattering measurements coincide with ELMs.

In conclusion, measurements of microwave and soft $\mathrm{x}$-ray emission during ELMs in spherical tokamak plasmas provide strong evidence for the transient presence in the edge plasma of highly suprathermal electrons. The paradoxical nature of the intensity of the emission, given the absence of any rf wave heating, is resolved by considering the response of electrons accelerated by a parallel electric field and invoking the collective anomalous Doppler instability. Particle-in-cell simulations show that magneticfield-aligned energetic electron distributions, of the kind inferred to result from parallel electric fields generated by ELMs, excite electromagnetic waves in the electron cyclotron range. Further, the PIC simulations predict emission frequencies between $\omega_{p e}$ and $\omega_{\mathrm{UH}}$; this is in turn consistent with the predictions from JOREK about the radial location of the accelerating field. While soft x-ray and Thomson scattering data indicate that the fraction of accelerated electrons is small, their active role suggests that purely fluid models of ELMs are incomplete. For example, the radial current associated with the rapid radial transport of these electrons could have an effect on ELM dynamics, and, if sufficiently energetic and present in sufficiently large numbers, they could cause damage to plasma-facing components. For these reasons it is hoped that the present study will prompt further investigations of energetic electron production during ELMs. Links have been drawn previously at the fluid level of description between ELMs and solar flares [14]; the present work suggests that these links may extend also to kinetic processes, specifically the production and evolution of energetic electron populations.

This project has received funding from the Research Councils UK Energy Programme [Grant No. EP/I501045], from the University of York, from the Engineering and Physical Sciences Research Council grant EP/H016732 and from Euratom. Assistance from Luca Garzotti and Rory Scannell on the interpretation of soft x-ray and Thomson scattering data is gratefully acknowledged. To obtain further information on the data and models underlying this paper, please contact PublicationsManager@ccfe.ac.uk. The views and opinions expressed herein do not necessarily reflect those of the European Commission.

[1] ASDEX Team, Nucl. Fusion 29, 1959 (1989).

[2] A. Loarte et al., Plasma Phys. Controlled Fusion 45, 1549 (2003).

[3] K. Kamiya et al., Plasma Phys. Controlled Fusion 49, S43 (2007). 
[4] G. Federici, A. Loarte, and G. Strohmayer, Plasma Phys. Controlled Fusion 45, 1523 (2003).

[5] G. T. A. Huysmans, S. Pamela, E. van der Plas, and P. Ramet, Plasma Phys. Controlled Fusion 51, 124012 (2009).

[6] B. Lloyd et al., Nucl. Fusion 51, 094013 (2011).

[7] C. H. Fuchs and M.E. Austin, Phys. Plasmas 8, 1594 (2001).

[8] G. Taylor, C. E. Bush, E. D. Fredrickson, H. K. Park, and A. T. Ramsey, Nucl. Fusion 32, 1867 (1992).

[9] M. E. Austin et al., Proceedings of the 14th Joint Workshop on ECE and ECRH, Santorini (Heliotopos Conferences Ltd, Athens, 2006).

[10] D. V. Bartlett et al., Proceedings of the 8th Joint Workshop on ECE and ECRH, Gut Ising (IPP Rep., Garching b. Munich, 1993).

[11] V. F. Shevchencko, R. G. L. Vann, S. J. Freethy, and B. K. Huang, JINST 7, P10016 (2012).

[12] B. Kurzan and K.-H. Steuer, Phys. Rev. E 55, 4608 (1997).

[13] H. A. S. Reid, Res. Astron. Astrophys. 14, 773 (2014).

[14] S. C. Cowley, H. Wilson, O. Hurricane, and B. Fong, Plasma Phys. Controlled Fusion 45, A31 (2003).
[15] Y. M. Wang, X. Gao, B. L. Ling, Y. Liu, S. B. Zhang, X. Han, A. Ti, E. Z. Li, and HT-7 Team, Phys. Plasmas 19, 032509 (2012).

[16] L. Vlahos and H. L. Rowland, Astron. Astrophys. 139, 263 (1984).

[17] E. Moghaddam-Taaheri and C. K. Goertz, Astrophys. J. 352, 361 (1990).

[18] C. Krafft and A. Volokitin, Ann. Geophys. 21, 1393 (2003).

[19] P. Testa et al., Science 346, 1255724 (2014).

[20] W. N. Lai, S. C. Chapman, and R. O. Dendy, Phys. Plasmas 20, 102122 (2013).

[21] A. K. Ram, A. Bers, and C. N. Lashmore-Davies, Phys. Plasmas 9, 409 (2002).

[22] A. Kirk, B. Koch, R. Scannell, H. Wilson, G. Counsell, J. Dowling, A. Herrmann, R. Martin, and M. Walsh, Phys. Rev. Lett. 96, 185001 (2006).

[23] S. J. P. Pamela, G. T. A. Huijsmans, A. Kirk, I. T. Chapman, J. R. Harrison, R. Scannell, A. J. Thornton, M. Becoulet, F. Orain, and the MAST Team, Plasma Phys. Controlled Fusion 55, 095001 (2013).

[24] A. B. Rechester and M. N. Rosenbluth, Phys. Rev. Lett. 40, 38 (1978).

[25] H. A. Kramers, Philos. Mag.Ser.6 46, 836 (1923). 